\title{
Modelling and Forecasting Volatility of Returns on the Ghana Stock Exchange Using Garch Models
}

\author{
${ }^{1}$ Frimpong Joseph Magnus and ${ }^{2}$ Oteng-Abayie Eric Fosu \\ ${ }^{1}$ Department of Finance and Accounting, KNUST School of Business, \\ Kwame Nkrumah University of Science and Technology, Ghana \\ ${ }^{2}$ Department of Economics and Statistics, School of Business, Garden City University College, Ghana
}

\begin{abstract}
This paper models and forecasts volatility (conditional variance) on the Ghana Stock Exchange using a random walk $(\mathrm{RW}), \operatorname{GARCH}(1,1), \operatorname{EGARCH}(1,1)$, and $\operatorname{TGARCH}(1,1)$ models. The unique 'three days a week' Databank Stock Index (DSI) was used to study the dynamics of the Ghana stock market volatility over a 10-year period. The competing volatility models were estimated and their specification and forecast performance compared with each other, using AIC and LL information criteria and BDS nonlinearity diagnostic checks. The DSI exhibits the stylized characteristics such as volatility clustering, leptokurtosis and asymmetry effects associated with stock market returns on more advanced stock markets. The random walk hypothesis is rejected for the DSI. Overall, the GARCH $(1,1)$ model outperformed the other models under the assumption that the innovations follow a normal distribution.
\end{abstract}

Keywords: Ghana Stock Exchange, developing financial markets, volatility, GARCH model

\section{INTRODUCTION}

Interest in financial markets and the possibility to forecast their course is connected to the growing recognition among economists, financial analysts, and policy makers of the increasing impact of financial variables on the macro economy and thus on economic policy in general. The Latin American, Southeast Asian, and Russian financial crises are good reminders of this fact. Also central to the interest in financial market variables is its importance in asset pricing, portfolio allocation, or market risk measurement. According to ${ }^{[1]}$ modelling volatility in financial markets is important because it sheds further light on the data generating process of the returns.

Financial sector development and reforms in many SubSaharan Africa (SSA) countries aimed at shifting their financial systems from one of bank-based to security market-based has orchestrated the establishment of many stock markets over the last two decades. Liberalisations and deregulations of markets for financial sector development to facilitate economic growth have also been informed by the drastic shift towards property-owning economies and the concomitant growing demand for access to capital ${ }^{[2]}$

As part of the financial sector reforms in Ghana, there have been renewed efforts aimed at promoting investments and listings on the Ghana stock market to open access to capital for corporate bodies and greater returns for investors. The stock market provides an added dimension of investment opportunity for both individuals and institutional investors with the fall in the returns on government treasury bills and bonds. Thus recent listings on the bourse saw equities being oversubscribed. See ${ }^{[3]}$ and ${ }^{[4]}$ for more stylized facts on the GSE. The current interest in understanding the dynamics of volatility of returns on the stock market by investors, markets practitioners, business press and academic researchers therefore, does not come as a surprise considering the rapid pace of development and change. Of particular interest to researchers is the ability to model and forecast future movements in returns based on information contained in historical trading activities.

The purpose of this study is to model and quantify volatility of returns on the Ghanaian stock market with different types of GARCH models. We use the basic random walk model, a symmetric GARCH $(1,1)$ model and two asymmetric EGARCH $(1,1)$ and TGARCH $(1,1)$ to capture the main characteristics of financial time series such as fat-tails, volatility clustering and the leverage effect. The basic random walk (RW) model is included to test for the random walk (Efficient Market) hypothesis. This information is clearly of particular importance for making economic decisions.

Corresponding Author: Frimpong Joseph Magnus Department of Finance and Accounting, KNUST School of Business, Kwame Nkrumah, University of Science and Technology, Ghana 
There is a large literature on modelling and forecasting volatility, however, none of such studies has appeared in the literature focusing on the Ghana Stock Exchange (GSE). ${ }^{[4]}$ used both daily and monthly stock data to examine calendar anomalies (day of the week and month of the year effects) in the GSE they employed non-linear models from the GARCH family in a rolling framework to investigate the role of asymmetries. Their evidence suggests that the best model is the threshold generalised autoregressive conditional heteroscedasticity (TGARCH) model. The TGARCH performs better than the OLS, GARCH, and EGARCH models in terms of both information criteria and the log likelihood function value for anomalies in rolling windows. According to ${ }^{[2]}$, studying African markets integration have suggested that the univariate EGARCH model suggested by ${ }^{[5]}$ are appropriate for the analysis of African market since they can successfully model asymmetric impacts of good news (market advances) and bad news (market retreats) on volatility transmission with high levels of accuracy. Using weekly market data from January 1993 to 2000 for Ghana, they found no evidence of asymmetry (i.e. leverage effect). This paper will focus on modelling and forecasting the conditional variance. ${ }^{[6]}$ for Egypt and ${ }^{[7]}$ for Kenya and Nigeria are other studies that focused on African markets.

The rest of this paper is organized as follows. Section 2 deals with the volatility models considered for this paper. The description of the DSI data and the methodology is presented in section 3 . The results and discussions are presented in section 4 and section 5 concludes the paper.

\section{Univariate Models of Conditional Volatility}

Random Walk Model: Traditional econometric models, such as the Ordinary Least Square method, are built on the assumption of constant variance. In the first place, supporters of the efficient market hypothesis (EMH) claim that stock price indices are basically random and as such any speculation based on past information is fruitless. The basic model for estimating volatility in stock returns using OLS is the naive random walk (RW) model is given in (1).

$$
r_{t}=\mu+\varepsilon_{t}
$$

where under the EMH or random walk hypothesis $\mu$, the mean value of returns, is expected to be insignificantly different from zero; and $\varepsilon_{t}$, the error term, should be not be serially correlated over time. Secondly, a more general AR1-OLS model could also be estimated as:

$$
r_{t}=\mu+\phi r_{t-1}+\varepsilon_{t}
$$

However, real-world financial time-series do not behave in a random manner. Financial time series, unlike other economic series, usually exhibit a set of peculiar characteristics. Stock market returns display volatility clustering or volatility pooling, where large changes in these returns series tend to be followed by large changes and small changes by small changes [8] leading to contiguous periods of volatility and stability. Stock returns also exhibit leptokurtosis, or in other words, the distribution of their returns tends to be fattailed [9] Yet another characteristic of stock returns is the exhibition of "leverage effect" which means that a fall in returns is accompanied by an increase in volatility greater than the volatility induced by an increase in returns ${ }^{[10]}$. These characteristics cannot be explained with linear models such as the RW and OLS. ${ }^{[11]}$ and ${ }^{[12]}$ independently introduced the autoregressive conditional heteroscedasticity $(\mathrm{ARCH})$ and the generalized ARCH models, which specifically allows for changing conditional variance. Various extensions of these GARCH models have been introduced to account for asymmetry effects. The basic GARCH $(1,1)$ variants derived below are estimated in this study. According to ${ }^{[13]}$, the lag order $(1,1)$ model is sufficient to capture all of the volatility clustering that is present from the data.

GARCH: The GARCH $(1,1)$ model by ${ }^{[12]}$ is based on the assumption that forecasts of time varying variance depend on the lagged variance of the asset. An unexpected increase or decrease in returns at time $t$ will generate an increase in the expected variability in the next period. The basic and most widespread model GARCH $(1,1)$ can be expressed as:

$$
\begin{aligned}
& r_{t}=\mu+\phi r_{t-1}+\varepsilon_{t} ; \varepsilon_{\mathrm{t}} / \Phi_{t-1} \sim N\left(0, h_{t}\right) \\
& h_{t}=\sigma+\alpha \varepsilon_{t-1}^{2}+\beta h_{t-1}
\end{aligned}
$$

where $\omega \succ 0, \alpha \succeq 0, \beta \succeq 0$. The $\operatorname{GARCH}(1,1)$ is weakly stationary if $\alpha+\beta \prec 1, \sigma$ is the mean, $\varepsilon_{t-1}^{2}$ is the news about volatility from the previous period (the ARCH term), and $h_{t-1}$ the conditional variance is the last period forecast variance (the GARCH term) and must be nonnegative.

The basic GARCH is symmetric and does not capture the asymmetry effect that is inherent in most stock markets return data also known as the "leverage effect". In the context of financial time series analysis the asymmetry effect refers to the characteristic of times series on asset prices that 'bad news' tends to increase volatility more than 'good news' ${ }^{[10,5]}$. The Exponential GARCH (EGARCH) model and the Threshold GARCH (TGARCH) model proposed by ${ }^{[5]}$ and ${ }^{[14]}$, respectively are specifically designed to capture the asymmetry shock to the conditional variance.

EGARCH: In the EGARCH model the natural logarithm of the conditional variance is allowed to vary 
over time as a function of the lagged error terms rather than lagged squared errors. The EGARCH $(1,1)$ model can be written as:

$$
\begin{aligned}
& r_{t}=\mu+\phi r_{t-1}+\varepsilon_{t} ; \varepsilon_{\mathrm{t}} / \Phi_{t-1} \sim N\left(0, h_{t}\right) \\
& \ln h_{t}^{2}=\varpi+\alpha\left|\frac{\varepsilon_{t-1}}{h_{t-1}}\right|+\gamma \frac{\varepsilon_{t-1}}{h_{t-1}}+\beta \ln h_{t-1}^{2}
\end{aligned}
$$

The exponential nature of the EGARCH ensures that the conditional variance is always positive even if the parameter values are negative, thus there is no need for parameter restrictions to impose nonnegativity. $\gamma$ captures the asymmetric effect.

TGARCH: The TGARCH modifies the original GARCH specification using a dummy variable. The TGARCH model is based on the assumption that unexpected changes in the market returns have different effects on the conditional variance of the returns. Good news goes with an unforeseen increase and hence will contribute to the variance through the coefficient $\beta$ instead of an unexpected decrease which is presented as a bad news and contributes to the variance with the coefficient $\alpha+\gamma$. If $\gamma>0$ the leverage effect exists and news impact is asymmetric if $\gamma \neq 0$. The GJR model is written as:

$$
\begin{aligned}
& r_{t}=\mu+\phi r_{t-1}+\varepsilon_{t} ; \varepsilon_{\mathrm{t}} / \Phi_{t-1} \sim N\left(0, h_{t}\right) \\
& h_{t}=\varpi+\alpha \varepsilon_{t-1}^{2}+\gamma \varepsilon_{t-1}^{2} \zeta_{t-1}+\beta h_{t-1} \\
& \text { where } \zeta_{t-1}=1 \text { if } \varepsilon_{\mathrm{t}-1}<0 \text { and } \zeta_{t-1}=0 \text { if } \varepsilon_{\mathrm{t}-1}>0
\end{aligned}
$$

\section{MATERIALS AND METHODS}

The sample of data used in this exercise is the daily closing prices of the Ghana Stock Exchange Databank Stock Index (DSI) over the period extending from 15 June 1994 to 28 April 2004 making total observations of 1508 excluding public holidays. The data are obtained from the Databank Research and Information Limited (DRIL), Ghana. The DSI was the first major share index on the GSE and its computation began on 12 November 1990. The index is composed of all the listed equities on the market. The DSI returns $\left(r_{t}\right)$ at time $t$ are defined in the natural logarithm of the DSI indices $(\mathrm{p})$, that is, $r_{t}=\ln \left(p_{t} / p_{t-1}\right)$. In order to make forecasts, the full sample is divided into two parts comprising 1342 in-sample observations from 15 June 1994 to 28 March 2003 and 166 out of sample observations from 31 March 2003 to 28 April 2004. Descriptive statistics for the DSI returns series are shown Table 1. As is expected for a time series of returns, the mean is close to zero.

Generally the index has a large difference between its maximum and minimum returns. The standard deviation is also high indicating a high level of
Table 1: Descriptive Statistics of DSI Returns Series

\begin{tabular}{lclc}
\hline Mean & 0.001961 & Skewness & 2.217609 \\
Max. & 0.121210 & Kurtosis & 44.86563 \\
Min. & -0.092106 & Jarque-Bera & 111439.8 \\
Std. Dev. & 0.010434 & Probability & 0.000000 \\
\hline
\end{tabular}

Sample: June 15, 1994 to April 28, 2004

fluctuations of the DSI daily return. There is also evidence of positive skewness, which means that the right tail is particularly extreme, an indication that the DSI has non-symmetric returns. DSI's returns are leptokurtic or fat-tailed, given its large kurtosis statistics in Table 1. The kurtosis exceeds the normal value of 3 . The series is non-normal according to the Jarque-Bera test, which rejects normality at the $1 \%$ level for each series. Figure 1 presents the patterns of the price and returns series of the DSI for the period under review. The index looks like a random walk. The density graph and QQ-plot against the normal distribution shows that the returns distribution also exhibits fat tails confirming the results in table1.

The autocorrelation coefficients of the squared DSI returns are presented in Table 2 . We can observe that the index shows evidence of ARCH effects judging from the significant autocorrelation coefficients. The significant autocorrelation in squared returns series proves the presence of volatility clustering that could be caused by the high kurtosis values. The autocorrelation in the series dies out after the 28 lags. The test $p$-values indicate a first order autocorrelation in the sample series (i.e. accepts the "no ARCH" hypothesis). The "no ARCH" hypothesis is however rejected for all other orders. This is an indication that an AR1 conditional mean model will be more suitable for our DSI series. Both the ADF and PP test statistics (not reported) reject the hypothesis of a unit root in the return series at $1 \%$ level of significance. These characteristics of the DSI returns series are consistent with other financial times series.

The RW, GARCH(1,1), EGARCH(1,1), TGARCH(1,1) models are estimated for the DSI returns series using the robust method of Bolleslev-Wooldridge's quasimaximum likelihood estimator (QMLE) assuming the Gaussian standard normal distribution. Next, we use a combination of information criteria such as minimum Akaike information criteria (AIC) and the maximum Log-likelihood (LL) values and a set of model diagnostic tests (ARCH-LM test, Q-statistics and BDS test) to choose the volatility model that best models the conditional variance of the DSI. For this exercise, the ${ }^{[15]}$ nonparametric BDS test for serial independence is applied. The test is used to detect non-linearity in the standardised residuals and is based on the null hypothesis that the data are pure white noise (completely random ${ }^{[16]}$. According to ${ }^{[17]}$, the principle is that once any linear or non-linear structure is 

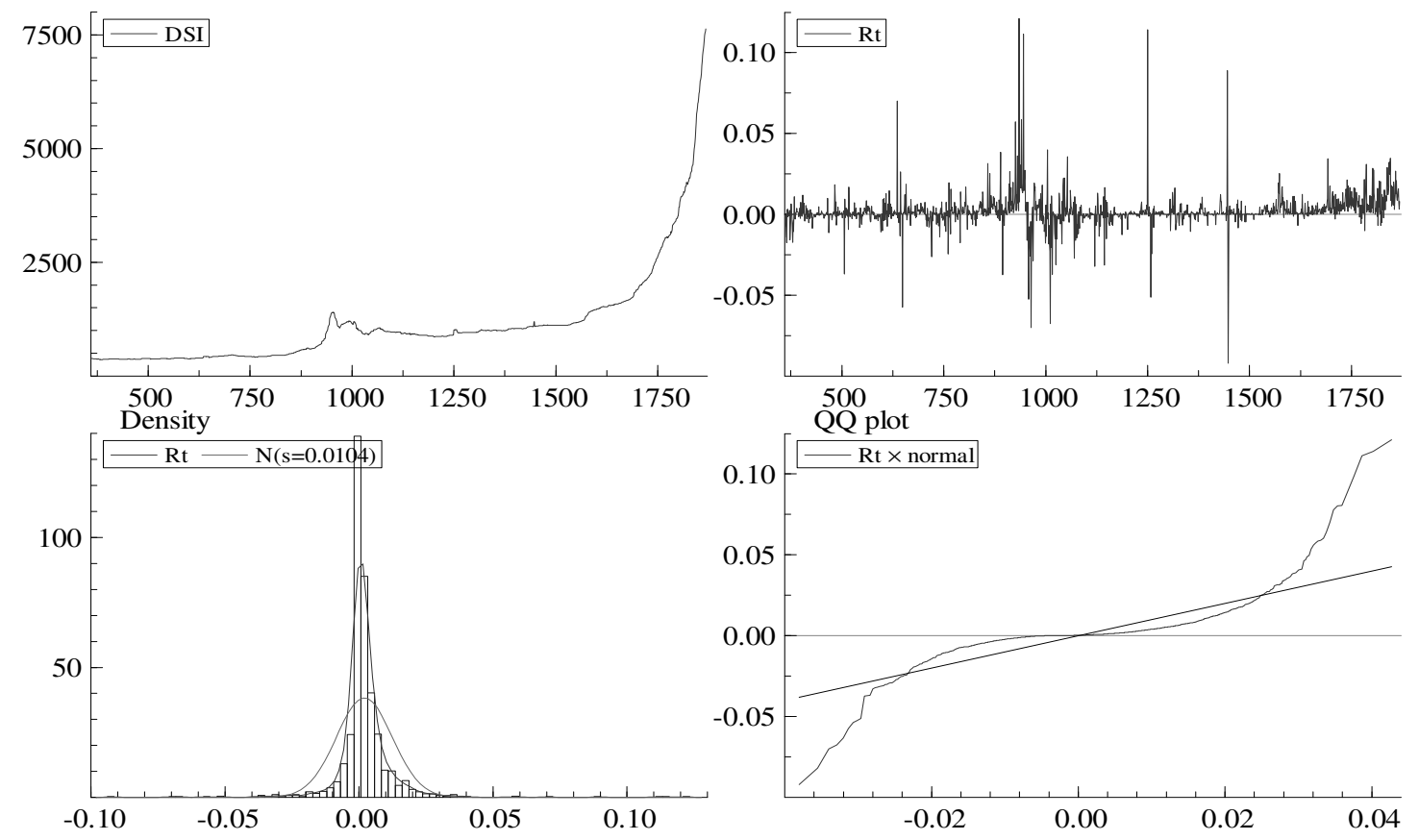

Fig. 1: DSI Returns and Tail Distribution

Table 2: Autocorrelation of Squared DSI Returns

\begin{tabular}{lllll}
\hline Order & AC & PAC & Q-Stat & p-value \\
\hline 1 & 0.018 & 0.018 & 0.4754 & 0.491 \\
3 & 0.019 & 0.016 & 18.878 & 0.000 \\
6 & 0.080 & 0.063 & 42.671 & 0.000 \\
9 & 0.068 & 0.058 & 56.747 & 0.000 \\
15 & 0.019 & -0.011 & 149.21 & 0.000 \\
21 & 0.003 & -0.020 & 173.53 & 0.000 \\
28 & 0.000 & -0.009 & 183.70 & 0.000
\end{tabular}

Sample: June 15, 1994 to April 28, 2004

Table 3: Estimated Volatility Models

\begin{tabular}{|c|c|c|c|c|}
\hline Models & RW & GARCH & TGARCH & EGARCH \\
\hline \multicolumn{5}{|c|}{ Mean Equation } \\
\hline$\mu$ & $0.0012(4.15)^{* * *}$ & $0.00059(2.95)^{* * *}$ & $0.00075(4.11)^{* * *}$ & $0.00039(1.37)$ \\
\hline$\phi$ & - & $0.16449(2.92)^{* * *}$ & $0.1163(2.92)^{* * *}$ & $0.05204(1.09)$ \\
\hline \multicolumn{5}{|c|}{ Variance equation } \\
\hline $\bar{\sigma}$ & - & $1.01 \mathrm{E}-05(1.31)$ & $6.50 \mathrm{E}-06(1.68)$ & $-0.62708(-1.61)$ \\
\hline$\alpha$ & - & $0.14208(3.40)^{* * *}$ & $0.15599(2.43)^{* *}$ & $0.14324(3.05)^{* * *}$ \\
\hline$\beta$ & - & $0.7798(10.34)^{* * *}$ & $0.85406(20.40)^{* * *}$ & $0.94170(23.40)^{* * * *}$ \\
\hline$\gamma$ & - & - & $-0.15418(-2.16)^{* *}$ & $0.12769(1.58)$ \\
\hline Q-stat (20) & $163.29 * * *$ & 1.4902 & 0.6235 & 0.7052 \\
\hline ARCH Test & $6.8442 * * *$ & 0.07228 & 0.03032 & 0.03399 \\
\hline AIC & $-6.2875^{4}$ & $-6.6397^{3}$ & $-6.6779^{2}$ & $-6.6887^{1}$ \\
\hline LL & $4219.90^{4}$ & $4460.26^{3}$ & $4486.89^{2}$ & $4494.10^{1}$ \\
\hline
\end{tabular}

Note: z-statistics [t-stat for RW] in brackets. $* * *(* *)$ denotes $1 \%(5 \%)$ significance. Superscripts ${ }^{(1)}$ denotes rank of model. 
Am. J. Applied Sci., 3 (10): 2042-2048, 2006

Table 4: BDS Diagnostic Tests for Serial Independence in Residuals

\begin{tabular}{|c|c|c|c|c|c|c|c|c|c|c|c|c|}
\hline & & RW & & & GARCH & & & TGARCH & & & EGARCH & \\
\hline \multicolumn{13}{|c|}{ BDS Asymptotic ( $p$-values) } \\
\hline Dimension & $\mathcal{E}=0.93$ & $\mathcal{E}=0.95$ & $\mathcal{E}=0.99$ & $\mathcal{E}=0.93$ & $\mathcal{E}=0.95$ & $\mathcal{E}=0.99$ & $\mathcal{E}=0.93$ & $\mathcal{E}=0.95$ & $\mathcal{E}=0.99$ & $\mathcal{E}=0.93$ & $\mathcal{E}=0.95$ & $\mathcal{E}=0.99$ \\
\hline 2 & 0.0 & 0.0807 & 0.9118 & 0.8901 & 0.5053 & 0.9351 & 0.4061 & 0.8425 & 0.9304 & 0.5829 & 0.6687 & 0.9078 \\
\hline 3 & 0.0 & 0.0010 & 0.9022 & 0.5157 & 0.8733 & 0.9096 & 0.0492 & 0.4664 & 0.9163 & 0.1444 & 0.6284 & 0.8827 \\
\hline 4 & 0.0 & 0.0005 & 0.8820 & 0.5571 & 0.8127 & 0.8899 & 0.0316 & 0.4431 & 0.8960 & 0.1261 & 0.5930 & 0.8615 \\
\hline 5 & 0.0 & 0.0000 & 0.8873 & 0.5920 & 0.7047 & 0.8680 & 0.0444 & 0.5465 & 0.8765 & 0.1508 & 0.6823 & 0.8403 \\
\hline \multicolumn{13}{|c|}{ BDS Bootstrap ( $p$-values) } \\
\hline 2 & 0.002 & 0.1640 & 0.850 & 0.9860 & 0.6960 & 0.3720 & 0.3760 & 0.8740 & 0.4440 & 0.5800 & 0.9300 & 0.9880 \\
\hline 3 & 0.002 & 0.0160 & 0.0660 & 0.4680 & 0.9860 & 0.4120 & 0.0820 & 0.3800 & 0.3960 & 0.1820 & 0.5700 & 0.9940 \\
\hline 4 & 0.002 & 0.0060 & 0.0940 & 0.5040 & 0.9600 & 0.4320 & 0.0500 & 0.3420 & 0.4540 & 0.1740 & 0.5680 & 0.9960 \\
\hline 5 & 0.000 & 0.0000 & 0.1260 & 0.5260 & 0.8040 & 0.5640 & 0.0500 & 0.4420 & 0.5160 & 0.1740 & 0.6420 & 0.8940 \\
\hline
\end{tabular}

Note: the ordinary residuals of the RW and standardized residuals of the GARCH models were used for the BDS test. Bootstrap with 1000 new sample and 1342 repetitions for all GARCH models. $\mathcal{E}$ denotes fraction of pairs epsilon value.

removed from the data, the remaining structure should be due to an unknown non-linear data generating process. In this case, if a model is the true data generating process then we expect its residuals to be white noise otherwise we reject the null hypothesis and consider the model as inadequate to capture all of the relevant features of the data. Hence the BDS test statistic for the standardised residuals will be statistically significant. We estimated the models using both EViews 5.1 and PcGive programs.

\section{RESULTS AND DISCUSSION}

The results of estimation and statistical verification of the RW, $\operatorname{GARCH}(1,1), \quad \operatorname{TGARCH}(1,1), \quad$ and EGARCH $(1,1)$ models are shown in Table 3 . The conditional mean $(\mu)$ and the AR1 $(\phi)$ parameters are significant in all the estimated models except the EGARCH model. The ARCH $(\alpha)$ and $\operatorname{GARCH}(\beta)$ terms are positive and significant in all estimations (Table 3). The sum of the ARCH and GARCH coefficients $(\alpha+\beta)$ is very close to one, indicating that volatility shocks are quite persistent.

The univariate RW, $\operatorname{GARCH}(1,1), \operatorname{TGARCH}(1,1)$ and $\operatorname{EGARCH}(1,1)$ models are used to provide estimates of the conditional volatilities associated with the DSI for the period 15 June 1994 to 28 April 2004. Table 3 reports the estimated parameters and the robust t-ratios.

Column 2 of table 3 reports the OLS estimate of the constant of the RW model in equation (1), together with a ARCH-LM test statistic. The results suggest that the mean $(\mu)$ of the return series is significantly different from zero, which is inconsistent with the random walk hypothesis. Furthermore, the battery of model diagnostic tests applied to the residuals of the RW model (Tables 3 and 4 ) are very significant at $1 \%$ level. ARCH-LM test and Q-stat of the standardized residuals show the presence of significant ARCH effects and autocorrelation in the RW model. The BDS test further strongly rejects the null hypothesis of a white noise (completely random) DSI returns at $1 \%$ significance level. The BDS result is also a suggestion of the absence of nonlinear dependence in the series. We therefore reject the hypothesis that the GSE - DSI follows a random walk. A comparison of the RW model with the other GARCH models using the minimum AIC and maximum LL values also indicates that it is the least preferred model to fit the DSI series.

The parameter estimates of all the GARCH models in table 3 show that the coefficients of the conditional variance equation, $\alpha$ and $\beta$, are significant at $1 \%$ and $5 \%$ levels implying a strong support for the ARCH and GARCH effects. The sum of the ARCH $(\alpha)$ and the $\operatorname{GARCH}(\beta)$ estimates are quite close to unity, which is an indication of a covariance stationary model with a high degree of persistence; and long memory in the conditional variance. In the $\operatorname{GARCH}(1,1)$ model, $\alpha+\beta=0.92188$ is also an estimation of the rate at which the response function decays on daily basis. Since the rate is high, the response function to shocks is likely to die slowly. In other words, if there is a new shock it will have implication on returns for a longer period. In such markets old information is more 
important than recent information and that the information decays very slowly. For the TGARCH and EGARCH models the persistence in volatility is very long and explosive suggestive of an integrated process. This is consistent with ${ }^{[4]}$. The asymmetric (leverage) effect captured by the parameter estimate $\gamma$ is negative and significant in the TGARCH suggesting the presence of a leverage effect. The asymmetry term is however positive and insignificantly different from zero in the EGARCH model, also suggesting no leverage effect. The presence of a leverage effect is mixed. The TGARCH results is consistent with ${ }^{[4]}$ for the same period under review while the EGARCH results is consistent with ${ }^{[2]}$ for the two separate periods (19932000) and (1997-2000) for the GSE stock index.

The results of the diagnostic tests show that the GARCH models are correctly specified. The Qstatistics for the standardized squared residuals are insignificant, suggesting the GARCH models are successful at modelling the serial correlation structure in the conditional means and conditional variances (Table 3). In the case of white noise (randomness) hypothesis, both the bootstrapped and asymptotic pvalues of the BDS test on the standardized residuals of all the GARCH models show that we can accept the null hypothesis of white noise (randomness) at 0.99 epsilon bound (Table 4).

Overall, using the minimum AIC, maximum LL values and the BDS test $p$-values as model selection criteria for the GARCH models, the preferred model is the EGARCH model based on the AIC and LL. However the BDS test results in table 4 shows that the $\operatorname{GARCH}(1,1)$ is the best model to capture all the serial dependence and inherent nonlinearity in the DSI returns.

Note: the ordinary residuals of the RW and standardized residuals of the GARCH models were used for the BDS test. Bootstrap with 1000 new sample and 1342 repetitions for all GARCH models. $\mathcal{E}$ denotes fraction of pairs epsilon value.

Forecast Performance: The models were also evaluated in terms of their forecasting ability of future returns. The common measures of forecast evaluation the RMSE, MAE, MAPE and TIC were used. In table 5 the results of the forecast of the performance are shown. The model that exhibits the lowest values of the error measurements is considered to be the best one. The results shows that the symmetric $\operatorname{GARCH}(1,1)$ model outperformed all the other models. This is supported by its highest $R^{2}$ value (not reported) compared to the others. The EGARCH(1,1) model performed the least in forecasting the conditional volatility of the DSI returns. These findings support ${ }^{[18]}$ view that relatively complex nonlinear models are inferior in forecasting to simpler parsimonious models. Figure 2 presents the outof-sample volatility forecast and variance forecast of the DSI returns.

\section{CONCLUSIONS}

The volatility of the DSI returns have been modelled for forecasting using a linear random walk model (RW), a nonlinear symmetric $\operatorname{GARCH}(1,1)$ model, and two nonlinear asymmetric models $\operatorname{TGARCH}(1,1)$ and $\operatorname{EGARCH}(1,1)$. We found that the DSI exhibits the stylised characteristics such as volatility clustering, leptokurtosis and asymmetry effects associated with stock returns on more advanced stock markets. The random walk hypothesis is also rejected for the GSE DSI returns. The parameter estimates of the GARCH models ( $\alpha$ and $\beta$ ) suggest a high degree persistent in the conditional volatility of stock returns on the Ghana Stock Exchange. The evidence of high volatility persistence and long memory in the GARCH models suggests that an integrated GARCH model may be more adequate to describe the DSI series. By and large, the $\operatorname{GARCH}(1,1)$ model is able to model and forecast the conditional volatility of the DSI better than the other competing models.

\section{REFERENCES}

1. Hongyu, P. and Zhichao, Z., 2006, "Forecasting Financial Volatility: Evidence from Chinese Stock Market." Working Paper in Economics and Finance, No. 06/02, University if Durham.

2. Piesse J and Hearn B 2002, Equity Market Integration versus Segmentation in Three Dominant Markets of the Southern African Customs Union: Cointegration and Causality Tests, Applied Economics, forthcoming

3. Osei, V., 2005, "Does the Stock Market Matter in Ghana? A Granger-Causality Analysis." Working Paper, WP/BOG-05/13, Bank of Ghana.

4. Alagidede P and Panagiotidis T, 2006. "Calendar Anomalies in an Emerging African Market: Evidence from the Ghana Stock Exchange," Discussion Paper Series 2006_13, Economics Department, Loughborough University, revised Jun 2006.

5. Nelson, D.B 1991 "Conditional Heteroscedasticity in Asset Returns: A New Approach', Econometrica 55, p 703-708. 
6. Eskandar, T., (2005), Modeling and Forecasting Egyptian Stock Market Volatility Before and After Price Limits. Working Paper \# 0310, The Economic Research Forum, September.

7. Ogum, G., Beer, F., and Nouyrigat, G., 2005, "Emerging Equity Market Volatility An Empirical Investigation of Markets on Kenya and Nigeria." Journal of African Business, 6: 1/2, 139 - 154, DOI: 10.1300/J156v06n01_08, ISSN: $1522-8916$.

8. Mandelbrot, B., 1963, "The Variation of Certain Speculative Prices", Journal of Business, 36, 394-419.

9. Fama, E., 1965, “The Behaviour of Stock Market Prices", Journal of Business38 (1), 34-105.

10. Panagiotidis, T., (2002), "Testing the Assumption of Linearity". Economics Bulletin, 3(29)1-9.

11. Dimson, E. and Marsh, P., 1990, "Volatility Forecasting without Data-Snooping". Journal of Banking and Finance, 14, $399-421$.
12. Bollerslev, T. 1986, Generalised Autoregressive Conditional Heteroskedasticity, Journal of Econometrics, 31, 307-27

13. Brooks, C. and Burke, S.P., 2003. Information Criteria for GARCH Model Selection: An Appplication to High Frequency Data, European Journal of Finance, 9:6, 557- 580.

14. Glosten, L. R; Jagannathan, R., and Runkle, D. E., 1993, "On the Relation between the Expected Value and the Volatility of the Nominal Excess Returns on Stocks." Journal of Finance, 48(5), 1779-1791.

15. Brooks Chris, Introductory econometrics for finance, first edition, Cambridge University press, Cambridge, 2002

16. Brock, W.A., Dechert, W. and Scheinkman, H and LeBaron, B. 1996, "A test for Independence Based on the Correlation Dimension". Econometric Reviews, 15, 197-235.

17. Panagiotidis, T., 2002, "Testing the Assumption of Linearity". Economics Bulletin, 3(29)1-9.

18. Dimson, E. and Marsh, P., 1990, "Volatility Forecasting without Data-Snooping". Journal of Banking and Finance, 14, $399-421$. 